\title{
Isolamento, identificação e avaliação da atividade antileucêmica de alcaloides indólicos monoterpênicos de Tabernaemontana salzmannii (A. DC.), Apocynaceae
}

\author{
Elaine R. Figueiredo, ${ }^{1}$ Ivo J. C. Vieira, ${ }^{, 2}$ Jucimar J. de Souza, ${ }^{2}$ Raimundo Braz-Filho, ${ }^{2}$ \\ Leda Mathias, ${ }^{2}$ Milton M. Kanashiro, ${ }^{3}$ Fernanda H. Côrtes ${ }^{3}$
}

\begin{abstract}
${ }^{1}$ Laboratório de Tecnologia de Alimentos, Universidade Estadual do Norte Fluminense Darcy Ribeiro, Av. Alberto Lamego, 2000, 28013-602 Parque Califórnia, Campos dos Goytacazes-RJ, Brasil, ${ }^{2}$ Laboratório de Ciências Químicas, Universidade Estadual do Norte Fluminense Darcy Ribeiro, Av. Alberto Lamego, 2000, 28013-602 Parque Califórnia, Campos dos Goytacazes-RJ, Brasil, ${ }^{3}$ Laboratório de Biologia do Reconhecer, Universidade Estadual do Norte Fluminense Darcy Ribeiro, Av. Alberto Lamego, 2000, 28013-602 Parque Califórnia, Campos dos Goytacazes-RJ, Brasil.
\end{abstract}

\begin{abstract}
RESUMO: O presente trabalho descreve o isolamento e a identificação de nove alcaloides indólicos monoterpênicos das cascas das raízes e folhas de Tabernaemontana salzmannii (Apocynaceae). As estruturas dos alcaloides foram identificadas através de métodos espectroscópicos uni (RMN ${ }^{1} \mathrm{H},{ }^{13} \mathrm{C}$, APT) e bidimensionais $\left({ }^{1} \mathrm{H}-{ }^{1} \mathrm{H}-\mathrm{COSY},{ }^{1} \mathrm{H}-{ }^{1} \mathrm{H}-\mathrm{NOESY}, \mathrm{HMQC}\right.$ e $\mathrm{HMBC}$ ) e espectrometria de massas (EM), além da comparação com dados de literatura. Um screening in vitro da atividade antileucêmica foi feito com os alcaloides majoritários isolados. Dentre os nove alcaloides isolados, a isovoacangina e voacangina mostraram-se capazes de induzir morte celular por apoptose em células leucêmicas humanas THP-1.
\end{abstract}

Unitermos: Tabernaemontana salzmannii, alcaloides indólicos, Apocynaceae, atividade antileucêmica.

\begin{abstract}
Isolation, identification and antileukemic activity of the monoterpene indole alkaloids from Tabernaemontana salzmannii (A. DC.), Apocynaceae". This work describes the isolation and structural determination of nine monoterpenic indole alkaloids from the roots bark and leaves of Tabernaemontana salzmannii. The alkaloids were identified by spectroscopic methods uni (NMR ${ }^{1} \mathrm{H},{ }^{13} \mathrm{C}$, APT) and two-dimensional $\left({ }^{1} \mathrm{H}-{ }^{1} \mathrm{H}-\mathrm{COSY},{ }^{1} \mathrm{H}-{ }^{1} \mathrm{H}-\mathrm{NOESY}, \mathrm{HMQC}\right.$ and HMBC) and mass spectra besides comparison with literature data. An in vitro screening was done with the isolated major alkaloids. Among the nine alkaloids isolated, isovoacangine and voacangine alkaloids were able to induce apoptosis cell death in human leukemic cells line THP-1.
\end{abstract}

Keywords: Tabernaemontana salzmannii, indole alkaloids, Apocynaceae, antileukemic activity.

\section{INTRODUÇÃO}

O gênero Tabernaemontana pertence à família Apocynaceae e em sua última revisão botânica feita por Leeuwenberg é reagrupado com outros gêneros, totalizando 99 espécies, das quais 27 são encontradas no Brasil (Leeuwenberg, 1994). A ocorrência de aproximadamente um quarto das espécies do gênero no Brasil demonstra a necessidade de aprofundar o estudo fitoquímico das espécies brasileiras do gênero.

Entre as apocináceas, Tabernaemontana é especialmenterico emalcaloides indólicos monoterpênicos, que são utilizados como marcadores químicos do gênero, sendo então usados na classificação de suas espécies (Van
Beek et al., 1984). Os alcaloides indólicos monoterpênicos apresentam inúmeras atividades biológicas, das quais se pode destacar as atividades, antitumoral, antimicrobiana, anti-hipertensiva e estimulante do sistema nervoso central (Verpoorte, 1998).

Em publicações anteriores relatamos o isolamento e identificação de alcaloides indólicos monoterpênicos das espécies T. laeta (Medeiros et al., 1999, 2001, 2003) e T. hystrix (Monnerat et al., 2005a), bem como a atividade anticolinesterásica de dezessete alcaloides indólicos (Vieira et al., 2008).

Como parte de nosso contínuo interesse na investigação fitoquímica de espécies de Tabernaemontana de ocorrência no Brasil, decidiu- 
se estudar Tabernaemontana salzmannii (A. DC.), Apocynaceae, uma espécie nativa da Mata Atlântica do sudeste do Brasil, popularmente conhecida como "espeta" sem relatos químicos na literatura.

T. salzmannii possui como sinonímia homotípico: Peschiera salzmannii (A. DC.) Miers, Apocyn. S. Am. 40 (1878) e sinonímia heterotípico: T. rauwolfiae A. DC. e T. salzmannii var. longifolia Müll. Arg. (Leeuwenberg, 1994).

No presente trabalho, é relatado o isolamento e identificação de nove alcaloides indólicos monoterpênicos das cascas das raízes e folhas de $T$. salzmannii, incluindo a atividade in vitro contra células leucêmicas humanas THP-1 dos alcaloides majoritários isolados.

\section{MATERIAL E MÉTODOS}

\section{Métodos gerais}

Separações cromatográficas em coluna aberta foram efetuadas em gel de sílica 60 G (70-230 e 230400 mesh, Merck) e $60 \mathrm{H}$ (Merck), ao passo que nas separações em camada delgada analítica e preparativa ( 0,25 e $1,0 \mathrm{~mm}$ de espessura, respectivamente) utilizou-se gel de sílica $60 \mathrm{PF}_{254}$ (Merck). As substâncias presentes foram visualizadas através de irradiação com lâmpada ultravioleta com comprimento de onda de $254 \mathrm{~nm}$ e 365 $\mathrm{nm}$ e/ou pulverizadas com reagente de Dragendorff e com solução de vanilina sulfúrica, seguidas de aquecimento. Os espectros na região do infravermelho foram registrados em pastilhas de $\mathrm{KBr}$ utilizando espectrofotômetro PerkinElmer, FTIR 1750. Os espectros de RMN ( ${ }^{1} \mathrm{H} 400 \mathrm{MHz}$; ${ }^{13} \mathrm{C} 100 \mathrm{MHz}$ ) foram obtidos em espectrômetro Jeol modelo Eclipse. O sinal residual do solvente ou o TMS foram utilizados como referência interna. Os deslocamentos químicos $(\delta)$ foram medidos em partes por milhão e as constantes de acoplamento $(J)$ foram medidas em Hz. Os espectros de massas foram registrados em um cromatógrafo a gás acoplado a um espectrômetro de massas Shimadzu, modelo CGMS-QP 5050 (IE, 70 eV, coluna capilar DB-5). A identificação dos alcaloides foi feita através da análise dos dados espectrométricos obtidos e por comparação com dados descritos em literatura.

\section{Material vegetal}

As cascas das raízes e folhas de Tabernaemontana salzmannii (A. DC.), Apocynaceae, foram coletadas pelo Prof. Dr. Ivo J. Curcino Vieira em agosto de 2000, nas proximidades da Reserva Biológica da Companhia Vale do Rio Doce, Linhares-ES, Brasil. A identificação botânica da espécie foi realizada pelo Botânico Domingos A. Folly, sua exsicata encontra-se depositada no herbário da Companhia com o código CVRD-133.

\section{Extração e isolamento dos constituintes químicos}

As cascas das raízes $(2,0 \mathrm{~kg})$ após secagem à temperatura ambiente e trituração, foram maceradas individualmente com os solventes hexano, diclorometano e metanol; as folhas $(1,2 \mathrm{~kg})$ foram submetidas a uma extraçãoácido/base $\left[\mathrm{H}_{3} \mathrm{PO}_{4}(4 \%) / \mathrm{NH} 4 \mathrm{OH}(10 \%)\right.$ eposterior extração com $\mathrm{CH}_{2} \mathrm{Cl}_{2}$ ]. Os extratos brutos das cascas das raízes em $n$-hexano (AH, 26,95 g), diclorometano (AD, 31,52 g), em metanol (AM, 14,81 g) e de folhas (AFD, $14,80 \mathrm{~g}$ ) foram obtidos após concentração das soluções em evaporador rotatório sob vácuo. Parte do extrato $\mathrm{AD}(20,0$ g) foi submetida a uma cromatografia em coluna com gel de sílica (230-400 mesh), utilizando como eluentes a mistura de solventes $\mathrm{CH}_{2} \mathrm{Cl}_{2}$ e $\mathrm{MeOH}$ em proporções crescente de polaridade e recolheram-se quinze frações. A fração $\mathrm{AD}-2$ $(3,5 \mathrm{~g})$ foi ressubmetidas à cromatografia utilizando a mesma mistura de solventes e recolheram-se sete frações. As frações AD-2-3 e AD-2-6 forneceram os alcaloides coronaridina $(\mathbf{1}, 400,0 \mathrm{mg})$ e 3-oxo-coronaridina $(\mathbf{9}, 12,0$ $\mathrm{mg})$, respectivamente. A fração AD-6 $(1,2 \mathrm{~g})$ foi novamente submetida à cromatografia utilizando a mesma mistura de solventes e recolheram-se quatro frações. A fração AD6-3 (521,0 mg) forneceu o alcaloide (19S)-heyneanina (2, $521,0 \mathrm{mg})$. Parte da fração AD-15 (50,0 mg) foi submetida à cromatografia em camada delgada preparativa, utilizando como eluentes a mistura de solventes $\mathrm{CH}_{2} \mathrm{Cl}_{2}: \mathrm{MeOH}$ 99:1 (v/v) fornecendo o alcaloide voachalotina (3, 5,0 $\mathrm{mg})$. O extrato AM (14,81 g) foi cromatografado em coluna com gel de sílica, utilizando misturas de solventes $\mathrm{CH}_{2} \mathrm{Cl}_{2}: \mathrm{MeOH}$ 99:1 (v/v) em proporções crescente de polaridade e recolheram cinco frações. A fração AM-2 foi recromatografada em coluna com gel de sílica, utilizando misturas de solventes $\mathrm{CH}_{2} \mathrm{Cl}_{2}$ e $\mathrm{MeOH}$ em proporções crescente de polaridade e recolheram seis frações. A fração AM-2-3 forneceu o alcaloide voacangina $(4,20,0 \mathrm{mg})$. A fração AM-5 foi recromatografada utilizando a mesma mistura de solventes e recolheram oito frações. A fração AM-5-3 forneceu o alcaloide olivacina $(\mathbf{8}, 8,0 \mathrm{mg})$. O extrato AFD (14,80 g), proveniente da extração ácido/base das folhas foi submetido à separação cromatográfica em coluna com gel de sílica, utilizando misturas de solventes $\mathrm{CH}_{2} \mathrm{Cl}_{2}$ e $\mathrm{MeOH}$ em proporções crescente de polaridade e recolheram quatorze frações. A fração AFD-4 (130,0 mg) forneceu o alcaloide isovoacangina $(\mathbf{5}, 130,0 \mathrm{mg})$. A fração AFD-5 (2,3 g) foi submetida à cromatografia utilizando a mesma mistura de solventes e recolheram quinze frações. A fração AFD-5-8 forneceu o alcaloide isovoacristina (6, $32,0 \mathrm{mg}$ ) e a fração AFD-5-10 forneceu o alcaloide (3S)hidroxiisovoacangina $(7,138,0 \mathrm{mg})$.

\section{Cultura de células leucêmicas THP-1}

A linhagem celular de origem mielóide leucêmica humana THP-1 foi cultivada em meio D-MEM F12 (Life Technology-USA) suplementado com $20 \mu \mathrm{g} / \mathrm{mL}$ de 
gentamicina (Life Technology-USA) e $10 \%$ de soro fetal bovino (Life Technology-USA). A cultura foi replicada a cada 2 d e mantida em estufa (Forma Scientific-USA) a $37{ }^{\circ} \mathrm{C}$, com $5 \%$ de $\mathrm{CO}_{2}$ e umidade relativa (Horn Jr et al., 2006).

Avaliação da atividade antileucêmica dos alcaloides (19S)-heyneanina (2), coronaridina (1), isovoacangina (5), isovoacristina (6) e voacangina (4)

As células leucêmicas humanas THP-1 foram semeadas em placas de 96 poços em volume de $200 \mu \mathrm{L}$ $\left(1,2 \times 10^{6}\right.$ cels $\left./ \mathrm{mL}\right)$ e tratadas com diferentes concentrações dos alcaloides e do medicamento antileucêmico vincrifil (sulfato de vincristina) e mantidas na estufa a $37^{\circ} \mathrm{C}$, com $5 \%$ de $\mathrm{CO}_{2}$ e umidade relativa. Nos tempos de 6,12 e $24 \mathrm{~h}$ as células foram coradas e as células apoptóticas, necróticas e normais foram contadas. Para o vincrifil (sulfato de vincristina), foram analisados os tempos de 12, 24, 36 e 48 h.

As células cultivadas com as diferentes drogas e em diferentes tempos foram coradas com uma solução de $1 \mu \mathrm{g} / \mathrm{mL}$ de laranja de acridina e $1 \mu \mathrm{g} / \mathrm{mL}$ de brometo de etidio. Após a montagem em lâmina e lamínula, as células foram levadas ao microscópio de fluorescência e contadas aproximadamente trezentas células em vários campos escolhidos aleatoriamente e discriminando-se as células apoptóticas, necróticas e normais, conforme descrito por Kosmider et al. (2004).

Resumidamente, o corante laranja de acridina penetra nas células com a membrana citoplasmática intacta e interage com o DNA emitindo fluorescência verde. Dessa forma, é possível diferenciar células morfologicamente normais de células com núcleos picnóticos, com a cromatina nuclear condensada e formação de corpos apoptóticos, características morfológicas de células em apoptose. As células em necrose são diferenciadas por serem permeáveis ao corante brometo de etidio, devido à perda da integridade da membrana plasmática, que interage com o DNA e emite fluorescência alaranjada.

\section{RESULTADOS E DISCUSSÃO}

\section{Identificação dos alcaloides}

A investigação fitoquímica de um espécime de Tabernaemontana salzmannii (A. DC.), Apocynaceae, permitiu o isolamento de nove alcaloides indólicos monoterpênicos 1-9. Estes alcaloides estão sendo descritos pela primeira vez na espécie, uma vez que não existem relatos na literatura sobre o estudo fitoquímico desta espécie. A caracterização destes alcaloides foi baseada na análise de dados espectrais, principalmente RMN uni-(1D: RMN ${ }^{1} \mathrm{H}, \mathrm{RMN}{ }^{13} \mathrm{C}-\mathrm{PND}$ e $\mathrm{RMN}{ }^{13} \mathrm{C}$-APT) e bidimensional [2D: ${ }^{1} \mathrm{H}-{ }^{1} \mathrm{H}-\mathrm{COSY},{ }^{1} \mathrm{H}-{ }^{1} \mathrm{H}-\mathrm{NOESY}, \mathrm{HMQC}$ e HMBC]. Os dados obtidos através destes espectros nos permitiram a atribuição inequívoca dos deslocamentos químicos de todos os átomos de hidrogênio $(\delta \mathrm{H})$ e carbono-13 $(\delta \mathrm{C})$.

Os sinais dos espectros de $\mathrm{RMN}{ }^{13} \mathrm{C}$ dos alcaloides 1-9 correspondentes a carbonos quaternários, metínicos, metilênicos e metílicos foram identificados pela análise comparativa dos espectros obtidos com desacoplamento de hidrogênio PND e com a técnica APT.

A atribuição inequívoca dos $\delta \mathrm{C}$ de todos os átomos de carbono foi assegurada pelas análises dos espectros bidimensionais de correlação heteronuclear HMQC e HMBC. A análise dos espectros bidimensionais de correlação homonuclear ${ }^{1} \mathrm{H}-{ }^{1} \mathrm{H}-\mathrm{COSY}$ permitiu reconhecer todas as interações spin-spin dos átomos de hidrogênio. Os espectros bidimensionais de interação dipolar ${ }^{1} \mathrm{H}-{ }^{1} \mathrm{H}-$ NOESY dos alcaloides 1-9 foram utilizados para confirmar as junções dos anéis e as conformações correspondentes.

Finalmente, o espectro de massas dos alcalóides 1-9, obtidos por impacto eletrônico a $70 \mathrm{eV}$, poderá ser útil também para investigações envolvendo o estudo de extratos brutos por CG/EM. A elucidação estrutural dos alcaloides 1-9 envolveu também a comparação com dados de literatura (Figueiredo, 2005; Monnerat, 2005b; Pereira et al., 2008; Souza, 2006).

Coronaridina (1): $\mathrm{RMN}^{1} \mathrm{H}\left(400 \mathrm{MHz}, \mathrm{CDCl}_{3}\right) \delta \mathrm{H}$ 7,91 (s, $\mathrm{HN}-1) ; 7,47$ ( $d, J=7,7 \mathrm{~Hz}, \mathrm{H}-9) ; 7,23(d, J=7,7 \mathrm{~Hz}$, $\mathrm{H}-12) ; 7,08(t, J=7,0 \mathrm{~Hz}, \mathrm{H}-10) ; 3,70\left(s, \mathrm{CO}_{2} \mathrm{CH}_{3}\right) ; 0,90$ $(t, J=7,3 \mathrm{~Hz}, \mathrm{H}-18) ; 1,88$ ( $s, \mathrm{H}-14) ; 1,33$ ( $m, \mathrm{H}-20) ; 3,57$ $(s l, \mathrm{H}-21) . \mathrm{RMN}{ }^{13} \mathrm{C}\left(100 \mathrm{MHz}, \mathrm{CDCl}_{3}\right): 135,47$ (C-2); 53,13 (C-3); 51,60 (C-5); 22,05 (C-6); 110,25 (C-7); 128,77 (C-8); 118,39 (C-9); 119, 17 (C-10); 121,87 (C-11); 110,32 (C-12); 136,56 (C-13); 27,34 (C-14); 32,01 (C-15); 55,06 (C-16); 36,46 (C-17); 11,61 (C-18); 26,72 (C-19); 39,09 (C-20); 57,43 (C-21); 175,70 (C-22); 52,51 (MeO22). $\mathrm{EM}(70 \mathrm{eV}) \mathrm{m} / z$ (int. rel.): 338 [M $\mathrm{M}^{+}$] (71), 253 (8), 214 (22), 148 (16), 136 (100), 122 (49).

(19S)-Heyneanina (2): RMN ${ }^{1} \mathrm{H}\left(400 \mathrm{MHz}, \mathrm{CDC}_{13}\right) \delta \mathrm{H}$ 7,80 ( $s, \mathrm{HN}-1) ; 7,47$ (dd, $J=8,1$ e 1,1 Hz, H-9); 7,26 (dd, $J=7,0$ e $2,9 \mathrm{~Hz}, \mathrm{H}-12)$; 7,16 ( $d t, J=7,0$ e $1,1 \mathrm{~Hz}, \mathrm{H}-11)$; $7,09(d t, J=8,1$ e $1,1 \mathrm{~Hz}, \mathrm{H}-10) ; 4,17(d q, J=6,2$ e 1,8 Hz, $\mathrm{H}-19) ; 3,85$ ( $s, \mathrm{H}-21) ; 3,73\left(s, \mathrm{CO}_{2} \mathrm{CH}_{3}\right) ; 1,11(d, J=6,2$ $\mathrm{Hz}, \mathrm{H}-18) ; 2,02$ ( $m, \mathrm{H}-14) ; 1,45$ ( $m, \mathrm{H}-20)$. RMN ${ }^{13} \mathrm{C}(100$ $\left.\mathrm{MHz}, \mathrm{CDCl}_{3}\right)$ : 135,52 (C-2); 51,17 (C-3); 52,19 (C-5); 21,46 (C-6); 109,79 (C-7); 128,47 (C-8); 118,41 (C-9); 119,43 (C-10); 122,24 (C-11); 110,44 (C-12); 135,72 (C-13); 26,76 (C-14); 22,91 (C-15); 54,06 (C-16); 36,95 (C-17); 20,33 (C-18); 71,35 (C-19); 39,53 (C-20); 59,77 (C-21); 174,88 (C-22); 52,32 (MeO-22). EM (70 eV) $\mathrm{m} / z$ (int. rel.): $354\left[\mathrm{M}^{+}\right]$(100), 336 (67), 309 (21), 214 (50), 152 (76), 154 (79), 130 (45).

Voachalotina (3): $\mathrm{RMN}{ }^{1} \mathrm{H}\left(400 \mathrm{MHz}, \mathrm{CDCl}_{3}\right) \delta \mathrm{H} 7,50(d$, $J=8,1 \mathrm{~Hz}, \mathrm{H}-9) ; 7,17$ ( $t, J=7,7 \mathrm{~Hz}, \mathrm{H}-10) ; 7,30(t, J=7,7$, $\mathrm{H}-11) ; 5,45$ ( $s, \mathrm{H}-19) ; 3,79\left(s, \mathrm{CO}_{2} \mathrm{CH}_{3}\right) ; 3,76(s, \mathrm{NCH} 3)$; $1,61(d, J=7,0 \mathrm{~Hz}, \mathrm{H}-18) ; 6,60(d, J=11,0 \mathrm{~Hz}, \mathrm{H}-3) ; 4,77$ 
$(d, J=6,2 \mathrm{~Hz}, \mathrm{H}-5) ; 3,19$ ( $m, \mathrm{H}-15) . \mathrm{RMN}{ }^{13} \mathrm{C}(100 \mathrm{MHz}$, $\mathrm{CDCl}_{3}$ ): 132,77 (C-2); 56,66 (C-3); 64,44 (C-5); 19,48 (C-6); 100,38 (C-7); 124,54 (C-8); 118,64 (C-9); 120,18 (C-10); 123,01 (C-11); 109,82 (C-12); 137,96 (C-13); 28,05 (C-14); 29,97 (C-15); 55,29 (C-16); 63,14 (C-17); 12,51 (C-18); 120,18 (C-19); 126,27 (C-20); 64,48 (C-21); 172,89 (C-22); 52,51 (MeO-22); 30,26 (MeN-1).

Voacangina (4): $\mathrm{RMN}{ }^{1} \mathrm{H}\left(400 \mathrm{MHz}, \mathrm{CDCl}_{3}\right) \delta \mathrm{H} 7,67(s$, $\mathrm{NH}) ; 7,13$ ( $d, J=8,8, \mathrm{H}-12) ; 6,92$ ( $d, J=2,6 \mathrm{~Hz}, \mathrm{H}-9)$; $6,80\left(d d, J=2,2\right.$ e 2,6 Hz, H-11); 3,85 ( $\left.\mathrm{sl}, \mathrm{OCH}_{3}\right) ; 3,71(\mathrm{sl}$, $\left.\mathrm{CO}_{2} \mathrm{CH}_{3}\right) ; 0,89(t, J=7,4 \mathrm{~Hz}, \mathrm{H}-18) ; 1,87$ ( $\left.s, \mathrm{H}-14\right) ; 1,31$ ( $m, \mathrm{H}-20) ; 3,54$ (sl, H-21). RMN ${ }^{13} \mathrm{C}\left(100 \mathrm{MHz} \mathrm{CDCl}_{3}\right)$ : 137,54 (C-2); 51,57 (C-3); 53,14 (C-5); 22,21 (C-6); 110,15 (C-7); 129,22 (C-8); 100,88 (C-9); 154,03 (C-10); 111,82 (C-11); 111,05 (C-12); 130,59 (C-13); 27,38 (C-14); 32,04 (C-15); 55,15 (C-16); 36,57 (C-17); 11,63 (C-18); 26,67 (C-19); 39,14 (C-20); 57,49 (C-21); 175,69 (C-22); 56,06 (MeO-10); 52,53 (MeO-22). EM (70 eV) $\mathrm{m} / z$ (int. rel.): $368\left[\mathrm{M}^{+}.\right]$(95), 353 (15), 244 (12), 184 (20), 160 (30), 122 (40), 152 (100).

Isovoacangina (5): $\mathrm{RMN}^{1} \mathrm{H}\left(400 \mathrm{MHz}, \mathrm{CDCl}_{3}\right) \delta \mathrm{H} 7,75(s$, $\mathrm{NH}) ; 6,76(d, J=2,2, \mathrm{~Hz}, \mathrm{H}-12) ; 7,33$ ( $d d, J=9,2$ e 2,6, Hz, $\mathrm{H}-9) ; 6,75$ ( $m, \mathrm{H}-10) ; 3,82\left(\mathrm{sl}, \mathrm{OCH}_{3}\right) ; 3,71\left(\mathrm{sl}, \mathrm{CO}_{2} \mathrm{CH}_{3}\right)$; $0,89(t, J=7,3, \mathrm{H}-18) ; 1,89(d, J=2,2 \mathrm{~Hz}, \mathrm{H}-14) ; 1,53$ ( $m, \mathrm{H}-20) ; 3,53$ (sl, H-21). RMN ${ }^{13} \mathrm{C}\left(100 \mathrm{MHz}, \mathrm{CDCl}_{3}\right)$ : 136,18 (C-2); 51,38 (C-3); 53,06 (C-5); 22,12 (C-6); 110,00 (C-7); 123,16 (C-8); 118,98 (C-9); 108,85 (C-10); 156,43 (C-11); 94,23 (C-12); 135,25 (C-13); 27,33 (C-14); 32,03 (C-15); 55,01 (C-16); 36,38 (C-17); 11,58 (C-18); 26,67 (C-19); 39,09 (C-20); 57,56 (C-21); 175,80 (C-22); 55,65 (MeO-11); 52,44 (MeO-22). EM (70 eV) $\mathrm{m} / z$ (int. rel.): $368\left[\mathrm{M}^{+}\right.$.] (90), 353 (7), 309 (6), 244 (12), 184 (30), 160 (25), 152 (70), 122 (35).

Isovoacristina (6): $\mathrm{RMN}{ }^{1} \mathrm{H}\left(400 \mathrm{MHz}, \mathrm{CDCl}_{3}\right) \delta \mathrm{H} 7,72$ $(s, \mathrm{NH}) ; 6,76$ ( $m, \mathrm{H}-12) ; 7,33(t, J=9,6 \mathrm{~Hz}, \mathrm{H}-9) ; 6,81(m$, $\mathrm{H}-10) ; 3,83\left(s, \mathrm{OCH}_{3}\right) ; 3,73\left(s, \mathrm{CO}_{2} \mathrm{CH}_{3}\right) ; 1,10(d, J=6,2$, $\mathrm{H}-18) ; 2,02$ ( $m, \mathrm{H}-14) ; 4,15$ (dq, $J=6,2$ e 1,8 Hz, H-19); $1,45$ ( $m, \mathrm{H}-20)$; 3,85 (s, H-21). RMN ${ }^{13} \mathrm{C}(100 \mathrm{MHz}$, $\left.\mathrm{CDCl}_{3}\right)$ : 134,36 (C-2); 51,03 (C-3); 52,18 (C-5); 21,56 (C-6); 109,67 (C-7); 122,88 (C-8); 119,10 (C-9); 109,25 (C-10); 156,78 (C-11); 94,32 (C-12); 136,28 (C-13); 26,80

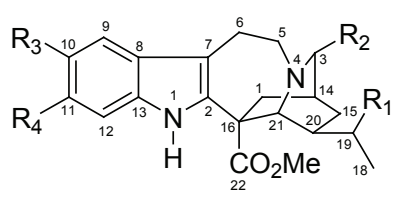

$1 \mathrm{R}_{1}=\mathrm{R}_{2}=\mathrm{R}_{3}=\mathrm{R}_{4}=\mathrm{H}$

$2 \mathrm{R}_{1}=\alpha \mathrm{OH} ; \mathrm{R}_{2}=\mathrm{R}_{3}=\mathrm{R}_{4}=\mathrm{H}$

$4 \mathrm{R}_{1}=\mathrm{R}_{2}=\mathrm{R}_{3}=\mathrm{H} ; \mathrm{R}_{4}=\mathrm{OMe}$

$5 \mathrm{R}_{1}=\mathrm{R}_{2}=\mathrm{R}_{4}=\mathrm{H} ; \mathrm{R}_{3}=\mathrm{OMe}$

$6 \mathrm{R}_{1}=\alpha \mathrm{OH} ; \mathrm{R}_{2}=\mathrm{R}_{3}=\mathrm{H} ; \mathrm{R}_{4}=\mathrm{OMe}$

$7 \mathrm{R}_{1}=\mathrm{R}_{4}=\mathrm{H} ; \mathrm{R}_{2}=\alpha \mathrm{OH} ; \mathrm{R}_{3}=\mathrm{OMe}$

$9 \mathrm{R}_{1}=\mathrm{R}_{3}=\mathrm{R}_{4}=\mathrm{H} ; \mathrm{R}_{2}=\mathrm{OMe}$
(C-14); 22,96 (C-15); 54,02 (C-16); 37,04 (C-17); 20,35 (C-18); 71,36 (C-19); 39,59 (C-20); 59,93 (C-21); 175,00 (C-22); 55,74 (MeO-11); 52,89 (MeO-22). EM (70 eV) $\mathrm{m} / \mathrm{z}$ (int. rel.): $384\left[\mathrm{M}^{+}\right.$.] (74), 366 (100), 339 (28), 244 (19), 184 (57), 174 (27), 152 (51), 140 (19).

(3S)-Hidroxiisovoacangina (7): $\mathrm{RMN}{ }^{1} \mathrm{H}(400 \mathrm{MHz}$, $\left.\mathrm{CDCl}_{3}\right) \delta_{\mathrm{H}} 7,86(s, \mathrm{NH}) ; 6,75(m, \mathrm{H}-12) ; 7,34(d, J=$ 9,2 Hz, H-9); 6,75 (m, H-10); 3,81 ( $\left.s, \mathrm{OCH}_{3}\right) ; 3,68(s$, $\left.\mathrm{CO}_{2} \mathrm{CH}_{3}\right) ; 0,92(t, J=7,3 \mathrm{~Hz}, \mathrm{H}-18) ; 4,41(s, \mathrm{H}-3) ; 1,88$ $(s l, \mathrm{H}-14) ; 3,75(s, \mathrm{H}-21) . \mathrm{RMN}{ }^{13} \mathrm{C}\left(100 \mathrm{MHz}, \mathrm{CDCl}_{3}\right)$ : 134,68 (C-2); 85,97 (C-3); 51,19 (C-5); 21,80 (C-6); 109,76 (C-7); 122,69 (C-8); 118,94 (C-9); 109, 10 (C-10); 156,55 (C-11); 94,32 (C-12); 136,26 (C-13); 35,42 (C-14); 24,72 (C-15); 54,43 (C-16); 35,42 (C-17); 11,61 (C-18); 26,83 (C-19); 37,73 (C-20); 56,26 (C-21); 174,94 (C-22); $55,70(\mathrm{MeO}-11) ; 52,58(\mathrm{MeO}-22)$. EM $(70 \mathrm{eV}) \mathrm{m} / z$ (int. rel.): $384\left[\mathrm{M}^{+}\right]$(26), 382 (100), 323 (35), 309 (15), 259 (28), 227 (43), 184 (61), 169 (31), 152 (34), 124 (31).

Olivacina (8): $\mathrm{RMN}{ }^{1} \mathrm{H}\left(400 \mathrm{MHz}, \mathrm{CDCl}_{3}\right) \delta \mathrm{H} \mathrm{3,03}(s$, H-21); 2,74 (s, H-17); 8,62 ( $s, \mathrm{H}-18) ; 8,19$ (d, $J=7,7 \mathrm{~Hz}$, H-9); 7,30 ( $m, \mathrm{H}-10$ e H-11); 7,26 ( $m, \mathrm{H}-12)$; 7,72 (d, $J=$ $6,2 \mathrm{~Hz}, \mathrm{H}-3) ; 7,51$ ( $d, J=5,5 \mathrm{~Hz}, \mathrm{H}-14) ; 2,74$ ( $s, \mathrm{H}-17)$. RMN ${ }^{13} \mathrm{C}\left(100 \mathrm{MHz}, \mathrm{CDCl}_{3}\right): 140,90$ (C-2); 137,93 (C-3); 122,17 (C-7); 123,05 (C-8); 120,88 (C-9); 119,45 (C-10); 127,63 (C-11); 110,63 (C-12); 142,33 (C-13); 115,14 (C-14); 132,87 (C-15); 110,94 (C-16); 11,96 (Me-17); 114,64 (C-18); 125,40 (C-19); 158,79 (C-20); 22,00 (Me21).

3-Oxo-coronaridina (9): $\mathrm{RMN}{ }^{1} \mathrm{H}\left(400 \mathrm{MHz}, \mathrm{CDCl}_{3}\right) \delta \mathrm{H}$ $7,47$ (d, $J=8,1 \mathrm{~Hz}, \mathrm{H}-9) ; 7,24$ (d, $J=8,1 \mathrm{~Hz}, \mathrm{H}-12) ; 7,09$ $(d t, \mathrm{H}-10) ; 7,14(d t, \mathrm{H}-11) ; 3,72\left(s, \mathrm{CO}_{2} \mathrm{CH}_{3}\right) ; 0,98(t, J=$ 7,4 Hz, H-18); 2,66 ( $m, \mathrm{H}-14) ; 2,63$ ( $m, \mathrm{H}-20) ; 4,49$ ( $m$, $\mathrm{H}-21) ; 3,21(m, \mathrm{H}-5) . \mathrm{RMN}{ }^{13} \mathrm{C}\left(100 \mathrm{MHz}, \mathrm{CDCl}_{3}\right): 133,93$ (C-2); 175,76 (C-3); 42,67 (C-5); 21,01 (C-6); 109,34 (C-7); 127,77 (C-8); 118,33 (C-9); 119,56 (C-10); 122,34 (C-11); 110,55 (C-12); 135,66 (C-13); 38, 13 (C-14); 30,95 (C-15); 55,51 (C-16); 35,84 (C-17); 11,31 (C-18); 27,58 (C-19); 35,43 (C-20); 56,08 (C-21); 173,03 (C-22); 52,99 (MeO-22). EM (70eV) m/z (int. rel.): 352 [M.$]$ (41), 229 (14), 180 (12), 154 (24), 124 (100), 81 (7).
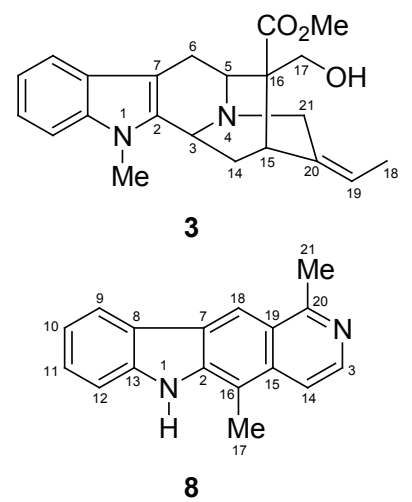


\section{Avaliação da atividade antileucêmica dos alcaloides majoritários}

A apoptose resulta do mecanismo de ação de várias drogas anti-neoplásicas, como por exemplo, o taxol e a cisplatina. Dessa forma, a capacidade de induzir apoptose é usada como um método de triagem de substâncias com ação antitumoral. Na Figura 1 podemos observar as alterações morfológicas, típicas de apoptose, das células THP-1 tratada com um alcaloide ativo. A laranja de acridina penetra nas células através da membrana intacta $\mathrm{e}$ interage com o DNA tornando-se espectralmente similar à fluoresceína fazendo que o núcleo emita uma fluorescência verde; de acordo com a morfologia nuclear são classificadas em células normais ou em apoptose. $\mathrm{O}$ brometo de etídio é capaz de penetrar apenas em células com membrana alterada e ao interagir com o DNA deixa o núcleo com uma coloração alaranjada fluorescente, caracterizando células necróticas ou com necrose secundária. As células em apoptose inicialmente apresentam o núcleo picnótico com as cromatinas condensadas e numa etapa mais avançada pode se observar a fragmentação nuclear formando corpos apoptóticos, Figura 1 (A e B). Por outro lado, as células normais apresentam a cromatina dispersa no núcleo e com morfologia característica da linhagem a que pertence, conforme Figura 1 (C e D).

Dentre os alcaloides majoritários testados [coronaridina (1), (19S)-heyneanina (2), isovoacangina (5), isovoacristina (6) e voacangina (4)] apenas a voacangina (4) e isovoacangina (5) apresentaram resultados satisfatórios de mortalidade celular (Tabela 1). Podese observar que a morte celular ocorreu em $100 \%$ pelo processo de apoptose. A voacangina (4) apresentou 80\% de apoptose na concentração de $100 \mu \mathrm{mol}$, num período de $24 \mathrm{~h}$, enquanto que a isovoacangina (5) apresentou $80 \%$ de células apoptóticas num período de $6 \mathrm{~h}$ na concentração de $100 \mu$ mol. Em todos os tempos estudados não foi observado número significativo de células coradas pela laranja de acridina o que sugere que nenhum dos alcalóides estudados foi capaz de induzir morte celular por necrose.

A semelhança estrutural entre as duas substâncias seria um ponto de partida para a avaliação da atividade, visto que ambas são substituídas no anel aromático por um grupo metoxila.

A isovoacristina (6) também possui a presença de um grupo metoxila, porém é hidroxilada no átomo de carbono $\mathrm{CH}-19$, informação esta, que pode ser útil em futuros estudos da relação da estrutura/atividade. Para um mesmo período de ação numa concentração de $50 \mu \mathrm{mol}$, por exemplo, a voacangina (4) num tempo de 24 h já apresentava $57,79 \%$ de morte por apoptose, para $29,51 \%$ na isovoacangina (5) e menos de $5 \%$ para isovoacristina (6) (dado não significativo). Os demais alcaloides não apresentaram atividade.

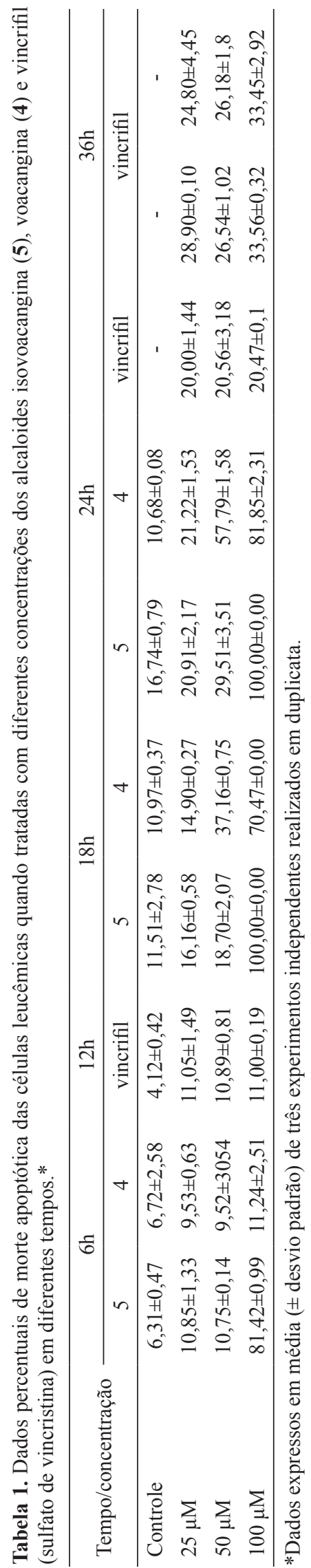


A

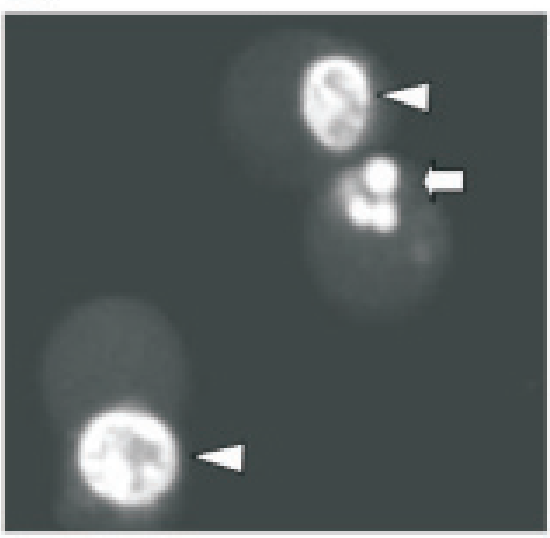

C

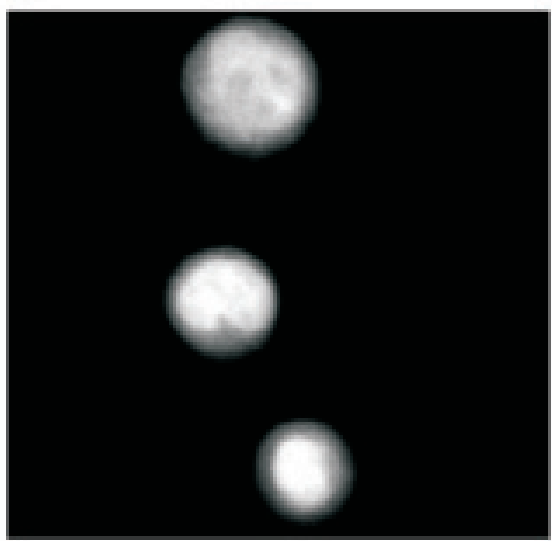

B

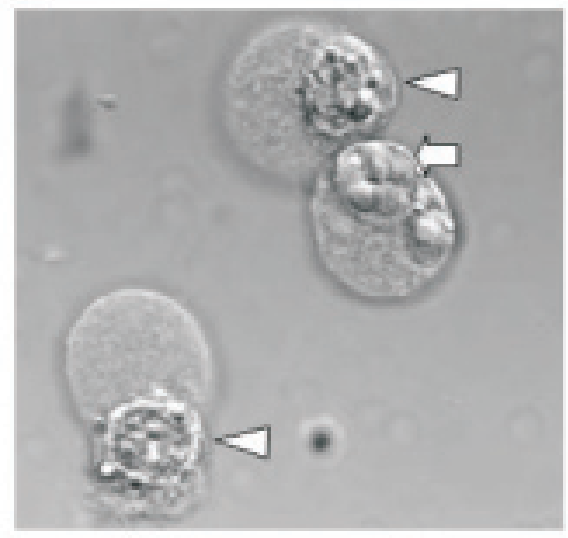

D

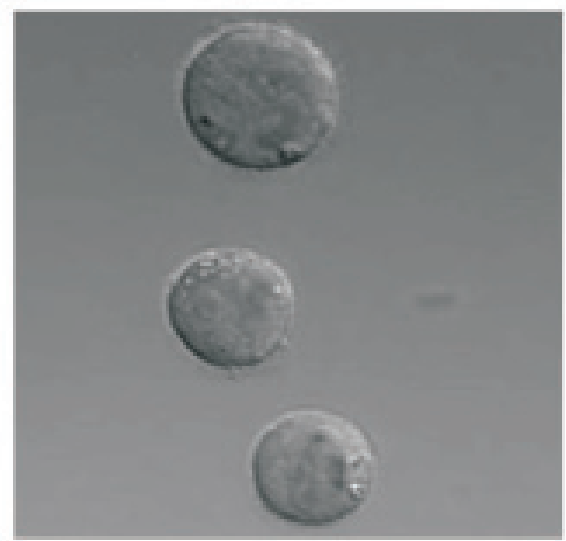

Figura 1. Avaliação das células THP-1 por microscopia de fluorescência (A e C), e por microscopia de contraste interferencial diferencial (B e D). A e B: Células THP-1 tratadas com voacangina (4) na concentração de $50 \mu \mathrm{Mol}$ e incubadas por 24 h; C e D: Controle; seta: corpos apoptóticos; cabeça de seta: célula com núcleo picnótico; aumento $400 \mathrm{x}$.

Tabela 2. Valores de IC50 representados em mM.*

\begin{tabular}{cccc}
\hline Alcaloide & $6 \mathrm{~h}$ & $18 \mathrm{~h}$ & $24 \mathrm{~h}$ \\
\hline 4 & & & \\
5 & $>100$ & 61,40 & 38,29 \\
vincrifil & $>10,16$ & 52,11 & 54,5 \\
\hline
\end{tabular}

*O cálculo de IC50 foi realizado utilizando-se o programa computacional Graphpad prism 5.0. Os dados para os cálculos foram obtidos da média de três experimentos independentes (Tabela 1).

Valores de $\mathrm{IC}_{50}$ foram estabelecidos para as três substâncias e expressos na Tabela 2. A isovoacangina (5) e a voacangina (4) apresentaram os valores de $\mathrm{IC}_{50}$ de 52,11 e $61,4 \mu \mathrm{Mol}$ em $18 \mathrm{~h}$ de incubação com as células leucêmicas THP-1, respectivamente. Sendo esses valores bem menores do que a concentração de sulfato de vincristina utilizado nesses experimentos. Esses dados são bastante interessantes e sinaliza a necessidade de uma avaliação mais detalhada destes dois alcaloides frente a outras linhagens celulares de origem leucêmica, a avaliação da toxicidade dos mesmos em células normais, bem como o estudo em modelos murinos de tumores e determinação da $\mathrm{DL}_{50}$.

Portanto, o estudo fitoquímico com os extratos das cascas das raízes e folhas de T. salzmannii conduziu ao isolamento de nove alcaloides indólicos monoterpênicos, sendo que destes, dois [voacangina (4) e isovoacangina (5)] mostraram-se bastante efetivos frente às células leucêmicas humanas da linhagem THP-1, sendo mais efetivos que o medicamento vincrifil (sulfato de vincristina) utilizado no tratamento de leucemia.

Os resultados obtidos neste estudo contribuem para um melhor conhecimento da química do gênero Tabernaemontana reforçando assim o interesse na investigação biológica com alcaloides indólicos monoterpênicos do gênero Tabernaemontana, dentro da família Apocynaceae, servindo também como ponto de partida para futuros estudos com outras linhagens de células cancerígenas, devido ao alto potencial 
antileucêmico apresentado pelos alcaloides voacangina (4) e isovoacangina (5).

\section{AGRADECIMENTOS}

Os autores agradecem as bolsas do $\mathrm{CNPq}$ e os apoios financeiros concedidos pelo Conselho Nacional de Desenvolvimento Científico e Tecnológico (CNPq), Coordenação de Pessoal de Nível Superior (CAPES), Fundação de Amparo à Pesquisa do Estado do Rio de Janeiro (FAPERJ) e a Universidade Estadual do Norte Fluminense Darcy Ribeiro.

\section{REFERÊNCIAS}

Figueiredo ER 2005. Alcaloides Indólicos de Tabernaemontana salzmannii. Campos dos Goytacazes, 218 p. Dissertação de Mestrado, Universidade Estadual do Norte Fluminense Darcy Ribeiro.

Horn A Jr, Fernandes C, Parrilha GL, Lessa JA, Santiago LJM, Kanashiro MM, Boniolo FS, Bortoluzzi AJ, Vugman NV, Herbst MH 2006. Synthesis, crystal structure, nuclease and in vitro antitumor activities of a new mononuclear copper (II) complex containing a tripodal $\mathrm{N}_{3} \mathrm{O}$ ligand. Inorg Chim Acta 359: 3167-3176.

Kosmider B, Zyner E, Osiecka R, Ochoki J 2004. Induction of apoptosis and necrosis in A549 cells by the cis-Pt(II) complex of 3-aminoflavone in comparison with cis-DDP. Mutat Res 563: 61-70.

Leeuwenberg AJM 1994. A Revision of Tabernaemontana. The new world species and Stemmadenia. UK: Royal Botanic Garden Kew.

Medeiros WLB, Vieira IJC, Leal KZ, Rodrigues-Filho E, Schripsema J 1999. Two know bis-indole alkaloids isolated from Tabernaemontana laeta: complete ${ }^{1} \mathrm{H}$ and ${ }^{13} \mathrm{C}$ chemical shift assignments. Magn Reson Chem 37: 676-681.

Medeiros WLB, Braz-Filho R, Vieira IJC, Mathias L, Schripsema $\mathrm{J} 2001$. A new natural quaternary indole alkaloid isolated from Tabernaemontana laeta Mart. (Apocynaceae). J Braz Chem Soc 12: 368-372.

Medeiros WLB, Vieira IJC, Mathias L, Braz-Filho R 2003. Complete ${ }^{1} \mathrm{H}$ and ${ }^{13} \mathrm{C}$ NMR chemical shift assignments of the bis-indole alkaloid tabernamine. Ann Magn Reson 1: 59-63.

Monnerat CS, Souza JJ, Mathias L, Braz-Filho R, Vieira IJC 2005a. A new indole alkaloid isolated from Tabernaemontana hystrix Steud. (Apocynaceae). J Braz Chem Soc 16: 1331-1335.

Monnerat CS 2005b. Alcaloides Indólicos de Tabernaemontana hystrix (Apocynaceae). Campos dos Goytacazes, 149 p. Dissertação Mestrado, Universidade Estadual do Norte Fluminense Darcy Ribeiro.

Pereira PS, França SC, Oliveira PVA, Breves CMS, Pereira SIV, Sampaio SV, Nomizo A, Dias DA 2008. Chemical constituents from Tabernaemontana catharinensis root bark: a brief NMR review of indole alkaloids and in vitro cytotoxicity. Quim Nova 31: 20-24.

Souza JJ de 2006. Constituintes químicos das cascas das raízes de Tabernaemontana hystrix (Apocynaceae). Campos dos Goytacazes, 96 p. Dissertação Mestrado, Universidade Estadual do Norte Fluminense Darcy Ribeiro.

Verpoorte R 1998. In: Roberts MF, Wink M (Ed). Alkaloids: Biochemistry, Ecology and Medicinal Applications. New York: Plenum Press.

Van Beek TA, Verpoorte R, Svendsen AB, Leeuwenberg AJM, Bisset NG 1984. Tabernaemontana L. (Apocynaceae): A review of its taxonomy, phytochemistry, ethnobotany and pharmacology. J Ethnopharmacol 10: 1-156.

Vieira IJC, Medeiros WLB, Monnerat CS, Souza JJ, Mathias L, Braz-Filho R, Pinto AC, Sousa PM, Rezende CM, Epifânio R 2008. A. Two fast screening methods (GC-MS and TLC-ChEI assay) for rapid evaluation of potential anticholinesterasic indole alkaloids in complex mixtures. An Acad Bras Cienc 80: 419-426. 